\title{
7
}

\section{EL PETRÓLEO EN EL ECUADOR: UN ANÁLISIS DEL COSTO DE AGOTAMIENTO, APLICACIÓN DEL MODELO HOTELLING.}

Víctor Solórzano Méndez, César Saltos Véliz, Mónica Célleri Zúñiga, Nancy Castillo Castro, Otto Vera Palacios 


\title{
EL PETRÓLEO EN EL ECUADOR: UN ANÁLISIS DEL COSTO DE AGOTAMIENTO, APLICACIÓN DEL MODELO HOTELLING \\ OIL IN ECUADOR: ANALYSIS OF THE EXHAUST COST, APPLICATION OF THE HOTELLING MODEL
}

\author{
Víctor Solórzano Méndez ${ }^{1}$; César Saltos Véliz, ${ }^{2}$; Mónica Célleri Zúñiga ${ }^{2}$; Nancy Castillo Castro ${ }^{2}$; Otto Vera \\ Palacios $^{1}$ \\ Universidad Estatal Península de Santa Elena ${ }^{1}$ \\ Universidad de Guayaquil $^{2}$ \\ vsolorza@gmail.com
}

\begin{abstract}
Resumen
El presente trabajo analiza el desarrollo de un pozo petrolero ecuatoriano, el cual estuvo en una senda sostenible durante el periodo 1975-1990 (análisis expost), a través del método de Hotelling, además del análisis de la explotación petrolera en el Ecuador en el periodo 2007-2015 y los cambios en políticas de inversión social, determinando que los dos períodos evaluados tienen incidencias sociales, económicas y ambientales que deben ser consideradas para investigaciones posteriores.
\end{abstract}

Palabras clave: desarrollo, petrolero, sostenible.

\section{Abstract}

The present work analyzes if the development of an Ecuadorian oil well was in a sustainable way during the period 1975-1990 (analysis expost), through the method of Hotelling in addition to the analysis of the oil exploitation in Ecuador in the period 2007-2015 And changes in social investment policies, determining that the two periods evaluated have social, economic and environmental impacts that should be considered for further research.

Keywords: development, petroleum, sustainable.

Recibido: octubre de 2016

Aprobado: diciembre de 2016 


\section{Introducción}

El Ecuador es un país rico en biodiversidad favorecido por su ubicación geográfica, posee dentro de sus recursos naturales el famoso "oro negro" es decir el petróleo, el cual tiene múltiples utilidades tanto como para su uso en el transporte, minerías e industrias, como fuente de energía. Pero al ser un recurso natural no renovable puede agotarse con el paso del tiempo.

Siendo un país primario exportador cuya dependencia principal es la explotación y exportación de petróleo, obtiene ingresos por la extracción sobre los 543 mil barriles por día en promedio (Ministerio de Hidrocarburos, 2016).En este sentido, el Presupuesto General del Estado de 2016, tiene como objetivo ampliar la cobertura del gasto social (educación, salud, viviendas, etc.)valiéndose de los ingresos petroleros.

$\mathrm{Su}$ precio se ha movido en los últimos cincuenta años en función de los depósitos o reservas que acumula Estados Unidos frente a una posibilidad de una conflagración, además del manejo político que tiene la Organización de Países Exportadores de Petróleo (OPEP), que en cualquier instante propone disminución de la oferta como lo plantearon en la reunión en Viena, realizada en el mes de noviembre de 2016; otro suceso que incide en el proceso de fluctuación del precio son los actos terroristas que ocurren permanentemente en los países del Oriente Medio.

Con las mejoras de los precios internacionales del barril de crudo, el Ecuador logró salir de la crisis ocurrida al finalizar el siglo XX, incluso en el 2008 en la problemática financiera internacional, el país no se vio afectado, lo que se evidencia en datos del Banco Central de Ecuador en los que el PIB a precios nominales creció en $6.4 \%$ con respecto al año anterior.

No obstante, el crecimiento económico no ha sido homogéneo, puesto que internamente en el país las desigualdades sociales se mantienen, las ciudades más grandes del país Quito, Guayaquil y Cuenca son las que mejor se han desarrollo.

Cuando se realiza la extracción del recurso natural, con el paso del tiempo se reduce el stock del capital natural, por lo que a futuro disminuirá los ingresos provenientes del petróleo. Por lo que evaluar el desarrollo sostenible es fundamental para economías como la del Ecuador, que utilizan un recurso natural para la obtención de ingresos, sobre todo cuando los niveles de extracción son mayores a las de inversión los cuales perjudicarán a las generaciones futuras.
Dicho esto, el bienestar de una economía depende de la venta del recurso natural, lo que se tiene que descubrir es si el precio de venta es el adecuado por la depreciación del capital natural (su desgaste), para esto es necesario entonces determinar el costo del agotamiento del petróleo, tomando el ejemplo de un pozo petrolero ecuatoriano.

El objetivo del presente estudio es determinar si el desarrollo de un pozo petrolero ecuatoriano estuvo en una senda sostenible durante el periodo 19751990 (análisis expost), aplicando el método de Hotelling para la explotación del petróleo.

\section{Evolución del proceso de extracción delpetróleo en el ecuador}

Los primeros indicios científicos de la existencia de petróleo en el Ecuador se registran el siglo pasado, que según varios autores, el primer pozo petrolero fue perforado en 1911 en la península de Santa Elena(Estrada, 2011)

En lo que se refiere a empresas que llegaron al país a la explotación, comercialización y refinación del denominado "oro negro" se menciona a la empresa Anglo en el año1922,la misma que operó en el país durante 67 años específicamente en la península de Santa Elena (Estrada, 2011)

En el año 1937, la empresa Shell en conjunto con EXXON, dan por terminada su operación petrolera en el país, aduciendo que los pozos situados en la Amazonía ecuatoriana no fueron productivos (Acosta, 2004).

En el año 1967, la empresa Texaco perforó el primer pozo comercial en la Amazonía. En los años siguientes, las mayores obras de infraestructura fueron el Sistema de Oleoducto Trans Ecuatoriano SOTE y la Vía Coca(Acosta, 2004).

Hasta el año 1990, Texaco operó el oleoductoy extrajo el $88 \%$ del total de la producción nacional de petróleo. Perforó 399 pozos y construyó 22 estaciones de perforación.

Hasta el año de 1971,miles de hectáreas habían sido entregadas a alrededor de media docena de empresas petroleras, sinestablecer regulaciones ni firmar contratos, en este mismo año el paísfue gobernado por una dictadura militar,la misma que decidió que el Ecuador formara parte delaOrganización de Países Exportadores de Petróleo (OPEP).

El 23 de junio de 1972, se creó la Corporación Estatal Petrolera Ecuatoriana (CEPE) y la primera exportación fue el 17 de agosto de 1972 con 308.238 barriles a \$2,34 el barril. (Velastegui, 2004).En el mismo año se puso en vigencia la Ley de 
Hidrocarburos, incrementándoselas regalías para el estado, se estableció que los contratos petroleros podían durar máximo 20 años y su extensión se fijó en 200.000 has., con lo cual las compañías devolvieron el $80 \%$ de las concesiones que le fueron otorgadas por 50 años (Velasteguí, 2004).

En el mes de septiembre del año de 1989 fue creada la empresa Petroecuador en reemplazo de CEPE y se creó un Holding, es decir, una matriz y seis filiales. Tres permanentes: Petroindustrial, Petroproducción y Petrocomercial; y tres temporales: Petropenínsula, Petroamazonas y Petrotransporte.

En 1993, por decisión del Arq. Sixto Durán Ballén, Presidente dela República del Ecuador, se retiró de la OPEP, reintegrándose en el período 2007-2008.

Desde 1985 hasta 1996 han existido 8 rondas petroleras que ocupan un área de aproximadamente 4.2 millones de hectáreas de las cuales casi 3.6 millones corresponden a los de los 13 millones de has., que conforman la Amazonia,ésta a su vez, representa el $46 \%$ del territorio nacional (Velastegui, 2004).

Cuando CEPE se hizo cargo de la petrolera los rendimientos bajaron; por tal motivo la Estatal Petroproducción entregó en 1996 a través de un contrato de prestación de servicios específicos hasta el 2017, los campos petroleros a la Escuela Superior Politécnica del Litoral (ESPOL). Esta a su vez, subcontrató a la argentina CGC (que se retiró en 2002) y finalmente contrató con Pacifpetrol.

En lo que se refiere a la novena ronda se produjo en 2002 donde se licitó los campos de la costa, excluyendo los de la Amazonía. Para fines del 2002 se termina de construir el Oleoducto de Crudos Pesados (OCP) como parte de la estrategia de expansión de la frontera petrolera (Velastegui, 2004).

Desde el año 2003, el Gobierno Nacional anuncia la décima ronda petrolera para la concesión de áreas en los territorios de Napo, Pastaza y Zamora Chinchipe, además de la continuación del proyecto ITT (Ishpingo, Tambococha, Tiputini) que está situado en el Parque Nacional Yasuní y la Reserva Faunística Cuyabeno. (Velasteguí, 2004)

En el 2006, se declaró la caducidad del contrato de explotación del bloque 15 que el Estado mantenía con la compañía Occidental. En ese año se alcanzaron precios records para el crudo a nivel mundial. (Acosta, 2004).En el territorio de la península de Santa Elena existen 2882 pozos petroleros y 1400 se encuentran en producción, con valores marginales.
Dentro del marco regulatorio en el año 2010, se publica la "Ley Reformatoria a la Ley de Hidrocarburos y a la Ley de Régimen Tributario Interno" en la que se determina que: En los contratos de prestación de servicios para la exploración y explotación de hidrocarburos los contratistas como operadores, no están sujetos al pago de regalías. La totalidad de la producción del área del contrato es de propiedad del Estado. (Baquero Daniel y Mieles José, 2015).

En cuanto a la prestación de servicios se determinó que, dentro de los ingresos provenientes de la producción de determinada área, el Estado ecuatoriano se reserva el $25 \%$ de los ingresos brutos como margen de soberanía. Estos cambios permitieron que para el año 2011 la producción nacional de petróleo de empresas públicas representara el $72 \%$ del total. En ese mismo año las revueltas suscitadas en los países árabes permitieron que el precio del crudo ecuatoriano aumentara en 35 $\%$ y llegara a $\$ 97$ por barril. (Baquero Daniel y Mieles José, 2015).

En el último año, el precio del barril de petróleo ha sufrido una brusca caída que ha complicado la economía ecuatoriana. El boom petrolero vivido en los últimos años permitió que el Gobierno Central realice una importante renovación de la infraestructura delpaís, sin embargo y debido a los constantes ciclos en el precio del petróleo, el Ecuadorse mantiene en una incertidumbre económica que espera una reactivación en otras áreas productivas y el aumento del precio del petróleo para estabilizar las finanzas nacionales. (Baquero Daniel y Mieles José, 2015).

El sistema de cuentas nacionales SCN realizado por las Naciones Unidas para homogenizar las cuentas macroeconómicas de todos los países del mundo ha tenido como base el cálculo del Producto Interno Bruto (PIB) desde la visión de crecimiento del indicador, no involucra el aporte de las mujeres en el área doméstica, no contabiliza tampoco la degradación ambiental, por lo tanto, no es un indicador efectivo para medir la depreciación de los recursos naturales.

Los gastos de protección del medio ambiente o gastos defensivos según la literatura económica del medio ambiente, en algunas ocasiones son cuantificados en otras no; lo que no hace un indicador de homogeneidad sobre comparaciones entre países; más aún, algunos países desarrollados llevan este proceso contable como son USA, Alemania y de América Latina: México, entre otros. 
Sobre el asunto de la contabilidad ambiental las propias Naciones Unidas han considerado que debe haber un registro contable sobre el daño ambiental denominadas cuentas satélites, que en el caso ecuatoriano no se aplican.

El 30 de julio de 1999 en el gobierno de Jamil Mahuad, con la promulgación de la Constitución del Ecuador en 1998, por primera vez se dicta una ley de gestión ambiental, en la cual en el Art. 5 estipula "Dispone que el Ministerio del Ambiente, por su parte, debe coordinar con los organismos competentes sistemas de control para la verificación del cumplimiento de las normas de calidad ambiental referentes al aire, agua, suelo, ruido, desechos y agentes contaminantes. Por otro lado, se establece que las obras públicas, privadas o mixtas y los proyectos de inversión públicos o privados que puedan causar impactos ambientales, deben previamente a su ejecución ser calificados, por los organismos descentralizados de control, conforme el Sistema Único de Manejo Ambiental.”

Esta ley de gestión ambiental fue lírica porque no se crearon los instrumentos burocráticos ni el financiamiento adecuado para su aplicación, tal es así que después en el año 2000 el país tuvoun proceso inestable hasta el año 2007, con el cambio presidencial cada dos años, por la inestabilidad democrática.

La nueva ley ambiental se la promulga en el año 2007 siendo Presidente de la República el Ec. Rafael Correa Delgado, se crea además el Ministerio del Medio Ambiente para precautelar y poder aplicar la ley. Entre las funciones más importantes de esta cartera de Estado se encuentran el cuidado de recursos hídricos y de los recursos forestales. Los gobiernos autónomos descentralizados (GAD) tienen competencias para crear ordenanzas para la protección del medio ambiente en sus límites territoriales como son: ruido, contaminación ambiental, recursos hídricos, control de residuos sólidos y líquidos, control de acuíferos y afluentes de agua.

\section{Estado del arte}

Los grupos conservacionistas de los Estados Unidos de Norteamérica, tuvieron su auge en medio de una eminente escasez de los recursos naturales que estaba ocurriendo en varios países desarrollados, esto obligó a la ciencia a buscar una tasa óptima de explotación que tuvo una primera propuesta a través del primer documento que se llamó "Recursos Agotable" por Harold Hotelling.

Esta regla manifiesta que, el precio de un bien agotable debe crecer a una tasa igual a la tasa de interés, esto dentro de una eficiencia en los procesos de extracción y un equilibrio competitivo dentro de la industria de cada recurso.Esto, en esencia, indica que el valor presente de un recurso agotable homogéneo debe ser idéntico sin importar el momento en el que ha sido extraído.

El óptimo de extracción y consumo de un recurso no renovable se encuentra en el punto de equilibrio de la función de Beneficios Marginales Sociales (BMS) y los Costos Marginales Sociales (CMS); pero analizando las situaciones sobre el uso del recurso, hoy implica un costo en el futuro o Costo de Oportunidad (CO) también llamado Costo de Uso (CU).Por lo que el CMS debe ser igual al Costo Marginal de Extracción (CMEx) más el Costo de Uso Marginal (CMU), donde la generación presente es mantenida por el coste de extracción; y, para las generaciones futuras es soportada por el coste de uso. La preocupación radica en que la extracción de los recursos actuales perjudicaría a las generaciones futuras, sin embargo si se deja de extraer,las generaciones actuales tendrían una pérdida de sus beneficios el día de hoy.Es por esto que se debe de considerar que los análisis de optimización del uso de un recurso no renovable deben ser intertemporales(Romero,1997).Considerando lo explicado por Romero, existen dos reglas para una mayor comprensión que son el principio de Hotelling(1931) y el principio de Hartwick (1977).

Al principio Hotelling pronostica que en un mercado perfectamente competitivo, el precio del mercado debe exceder el costo marginal por la diferencia de la renta adicional que es ocasionada por la escasez del recurso natural.Para los recursos naturales no renovables como el petróleo, será necesario realizar estimaciones con modelos dinámicos, y de esta manera los resultados serán más precisos al considerar los efectos mencionados.

A raíz de la publicación de Hotelling; y, basándonos en las diferentes variedades de recursos que cumplen estas características, varios académicos han explorado empíricamente tanto el modelo original como otras versiones y sus respectivos ajustes según el tipo de mercado, entre ellos Hartwick. Quien define que la producción no solo depende de la tasa de consumo del recurso o también llamado capital natural, sino también del stock de capital disponible hecho por el hombre, demostrando que dada la condición de eficiencia local de Hotelling, una inversión agregada neta con valor de cero implicará consumo constante a través del tiempo, lo que finalmente se llamó el principio de Hartwick(Asheim \& Buchholz, 2000). 
El hallazgo de Hartwick conlleva que el capital natural puede ser reemplazado por el capital hecho por el hombre, por lo que el consumo y agotamiento de un recurso natural no ocasionaría ningún tipo de daño a las generaciones futuras.Es por esto que se consideran políticas de inversión para generar sostenibilidad en el tiempo y de esta manera se da lugar a las regalías por la extracción del recurso.Siendo un beneficio para el Estado quien recibe un valor por la producción del recurso natural, y no por la exploración; teóricamente hablando dentro de un mercado competitivo, las regalías por unidad producida del recurso natural deben ser iguales al costo de uso marginal del mismo para cumplir con las condiciones de optimización(Daly\& Farley, 2004).

El principio de Hartwick también dio lugar al concepto de sostenibilidad débil, donde las diferentes formas del capital serán sustituibles por las realizadas por el hombre y su valor se mantendrá constante (Harwick \& Olewiler, 1998); por lo que el capital natural se puede agotar y las generaciones futuras no se verán afectadas. Algunos economistas consideran que no es aceptable la sustitución de ciertos recursos naturales y que con el paso del tiempo, de igual manera se verán afectadas las generaciones futuras.Los ecologistas a su vez indican que esto implicaría un efecto negativo.Por lo que se debe considerar un mínimo de stock para prevenir catástrofes a nivel mundial los cuales deben de mantenerse con el paso del tiempo, esto se conoce como sostenibilidad fuerte (Perman, MacGilvary, \& Common, 1999), concepto que se contrapone a lo expuesto anteriormente.

Hotelling también planteó su modelo bajo un supuesto de monopolio; donde el principio del comportamiento del precio se cumplía, pero el ingreso marginal se volvía igual al precio de sustitución. También señalaba que la existencia de monopolio y otras fallas de mercado promueven alteraciones de los precios, de tal forma que se genera una tasa de producción menor a la óptima, y de que esa manera se presiona un alza en los precios y quien paga eso es el consumidor.El propietario del recurso agotable busca maximizar el valor presente.

En el caso de Ecuador, hay muy pocos estudios sobre el agotamiento del petróleo; donde la mayor problemática se halla en la riqueza de los recursos naturales sean estos renovables o no; por su abundancia se cambia de la extracción de un recurso primario a otro recurso primario, convirtiendo al país en mono-productor o primario-exportador. Lo que lo convierte en un país dependiente de los precios del mercado internacional.
Al no tener un ejercicio empírico para el caso del petróleo, y dado el comportamiento de los últimos años, donde se tiene un alza en el precio del petróleo lo que provoca una mayor extracción del recurso natural para generar más ingresos; y a su vez la disminución del mismo que ocasiona también una mayor extracción; pero esta vez, para tratar de cubrir los gastos ya pactados.Este incremento de producción provoca la reducción de las reservas por lo que la estimación del costo de uso es de suma importancia para el análisis de las políticas ecuatorianas.

\section{Consideraciones estadísticas de la producción de petróleo}

La industria petrolera es administrada en un alto porcentaje por el propio Estado, por lo cual actúa como un mercado monopolístico, las fluctuaciones del precio del barril del petróleo se basan no a un mercado competitivo, ni a un mercado monopolístico; sino que se ve afectado a causa de los carteles en el mercado internacional. Como muestra,en el año 2014, Estados Unidos disminuyó su demanda petrolera, y China también lo hizo debido a su ralentización económica.

El Ecuador, en el último milenio, ha mejorado su capacidad de refinación de los derivados: gasolina, diesel y otros. Desde inicios del 2014 ha sido autosuficiente, solamente en el mes de septiembre a diciembre del 2014 se tuvo un desabastecimiento interno teniendo que importar más derivados por cuanto la refinería de Esmeraldas entró en un proceso de revisión de su planta, en el año 2007 se exporta 186.67 millones de barriles de petróleo, en el 2015 fue de 198.23 millones de barriles de petróleo, con una tasa de crecimiento en el periodo analizado de la exportación petrolera que alcanzó a $0.86 \%$, menor que el $1 \%$.

La extracción en el sector privado, en el periodo analizado, tiene un proceso de decrecimiento debido a que muchas empresas extranjeras se retiraron en este periodo de análisis, favoreciendo al sector estatal, permitiendo una mayor entrada de divisas para el gobierno nacional que se vio reflejado en el aumento del capital natural: obra, infraestructura, puente,y además también en el capital humano, (formación de profesores de escuelas, colegios, universidades, becas de estudio en todos los niveles).

Es de resaltar que la inversión pública en el área hidrocarburífera se ha multiplicado por 6 en el periodo analizado, como la repotenciación de la refinería de Esmeraldas. Además, la exploración en el campo Yasuní ITT, y los otros campos petrolíferos del Oriente ecuatoriano. 
Al ser favorecido por el incremento del precio del petróleo que superó la barrera de los $\$ 100$ entre el 2014-2015, permitió mejorar e invertir mucho más en el área de exploración y explotación petrolífera, pero a finales del 2015, la caída del precio del petróleo se ve reflejada con una disminución de las inversiones petrolíferas debido a que el precio llegó a ser menor al precio de extracción.

Recién para septiembre y octubre de 2016, el precio del barril está alcanzando más de $\$ 50$ debido a que la OPEP Rusia determinó disminuir la oferta de petróleo diaria, que alcanza los 30 millones de barriles diarios.

La inversión pública por la exploración del petróleo se ha incrementado a partir del año 2007, yendo de la mano con el incremento del precio del barril del petróleo, generando plazas de empleo para investigación y extracción, mientras que a finales del 2014 y 2015 las inversiones empiezan a decaer generando desbalances financieros que perjudican la economía nacional.

\section{Metodología de la valoración de los recursos naturales.}

La economía neoclásica jamás planteó la depreciación de los recursos naturales: bosques, minas, clima, recursos hídricos, recursos marítimos, recursos oceánicos, etc.En el proceso de producción de bienes y servicios para satisfacer las necesidades de la sociedad se utilizan mayoritariamente recursos naturales; que, tienen que ser considerados su proceso de agotamiento y debe ser vinculante en la contabilidad nacional que lamentablemente los países en vías de desarrollo, caso Ecuador, no contemplan este mecanismo y sólo consideran el Producto Interno Bruto (PIB) como el indicador de crecimiento.

Para el caso del pozo ecuatoriano, se aplicó la propuesta de Hotelling para un pozo específico, la senda de extracción y su agotamiento vinculado con lajError! No se encuentra el origen de la referencia.

Tabla 1: producción petrolera pozo A0045

\begin{tabular}{|c|c|c|c|c|c|c|c|c|}
\hline Año & $\begin{array}{c}\text { Precio } \\
\text { exportación } \\
\text { del petróleo }\end{array}$ & $\begin{array}{c}\text { Cost } \\
0\end{array}$ & Renta & $\begin{array}{c}\text { Tipo de } \\
\text { cambio } \\
\text { Sucres por } \\
\text { US\$ }\end{array}$ & $\begin{array}{c}\text { Producción } \\
\text { Pozo A0045 } \\
\text { (BDP } \\
\text { promedio) }\end{array}$ & $\begin{array}{c}\text { Total } \\
\text { barriles }\end{array}$ & Beneficios & $\begin{array}{c}\text { Beneficios } \\
\text { descontados }\end{array}$ \\
\hline \multicolumn{9}{|c|}{ US\$ por barril } \\
\hline 1975 & 11.5 & 0.61 & 10.89 & 25 & 114 & 37620.0 & $\$ 409,681.80$ & $\$ 365,787.32$ \\
\hline 1976 & 11.5 & 0.63 & 10.87 & 25 & 114 & 37620.0 & $\$ 408,929.40$ & $\$ 325,996.01$ \\
\hline 1977 & 13 & 0.65 & 12.35 & 25 & 100 & 33000.0 & $\$ 407,550.00$ & $\$ 290,086.04$ \\
\hline 1978 & 12.5 & 0.67 & 11.83 & 25 & 90.00 & 29700.0 & $\$ 351,351.00$ & $\$ 223,289.91$ \\
\hline 1979 & 23.5 & 0.87 & 22.63 & 25 & 85.50 & 28215.0 & $\$ 638,505.45$ & $\$ 362,305.14$ \\
\hline 1980 & 35.26 & 1.02 & 34.24 & 25 & 82.08 & 27086.4 & $\$ 927,438.34$ & $\$ 469,869.12$ \\
\hline 1981 & 34.48 & 1.29 & 33.19 & 25 & 73.87 & 24377.8 & $\$ 809,097.85$ & $\$ 365,994.78$ \\
\hline 1982 & 32.84 & 2.15 & 30.69 & 31 & 62.79 & 20721.1 & $\$ 635,930.44$ & $\$ 256,841.64$ \\
\hline 1983 & 38.08 & 3.61 & 34.47 & 50 & 54.44 & 17965.2 & $\$ 619,260.11$ & $\$ 223,311.40$ \\
\hline 1984 & 27.46 & 3.7 & 23.76 & 71 & 46.38 & 15306.3 & $\$ 363,678.69$ & $\$ 117,094.80$ \\
\hline 1985 & 25.9 & 4.32 & 21.58 & 93 & 38.82 & 12811.4 & $\$ 276,470.19$ & $\$ 79,478.57$ \\
\hline 1986 & 12.78 & 4.74 & 8.04 & 132 & 31.91 & 10531.0 & $\$ 84,669.06$ & $\$ 21,732.44$ \\
\hline 1987 & 16.28 & 5.68 & 10.6 & 190 & 27.13 & 8951.3 & $\$ 94,884.11$ & $\$ 21,744.99$ \\
\hline 1988 & 12.68 & 4.27 & 8.41 & 331 & 25.77 & 8503.8 & $\$ 71,516.66$ & $\$ 14,633.73$ \\
\hline 1989 & 16.2 & 3.52 & 12.68 & 532 & 23.19 & 7653.4 & $\$ 97,044.96$ & $\$ 17,729.75$ \\
\hline \multirow[t]{2}{*}{1990} & 20.36 & 2.06 & 18.3 & 776 & 20.87 & 6888.0 & $\$ 126,051.30$ & $\$ 20,561.70$ \\
\hline & & & & $\begin{array}{l}\text { Reserva } \\
\text { probada }\end{array}$ & 990.76 & 326950.7 & & $\$ 3,176,457.35$ \\
\hline
\end{tabular}

Fuente: datos sobre información petrolera del Ecuador Elaboración: autores

La metodología se apoyará en el modelo formulado por Hotelling, donde existe la aplicación de sus modelos teóricos en el cual, mediante el levantamiento de información secundaria, se obtuvo información de datos históricos de un pozo petrolero agotado, considerando como limitante ya que no existe información oficial con respecto a los costos de extracción. 
El modelo de Hotelling busca determinar los senderos óptimos de extracción de un recurso no renovable, para este caso el recurso sería el petróleo, considerando las siguientes variables:

P Precio del recurso (En este caso del petróleo)

C Costo por barril de petróleo

Q Cantidad de producción ( Se tiene valores tanto diarios como anuales)

Tc Tipo de cambio Sucres por US\$

\section{B Beneficios}

El objetivo de la renta de Hotelling es maximizar los beneficios por el uso de los recursos naturales no renovables, para este trabajo se ha utilizado el modelo monopólico, puesto que el Estado es dueño del petróleo, cuyos ingresos obtenidos por cada barril es utilizado en la actualidad para la inversión pública como lo refleja la Tabla 3.
En el caso del petróleo, el Estado maximiza los beneficios cuando el ingreso marginal (I') iguala al costo marginal (C') por la extracción de cada barril, siendo $I^{\prime}=\frac{d I}{d q} \mathrm{y} C^{\prime}=\frac{d C}{d q}($ Orihuela, 2016); es decir:

$$
I^{\prime}(t)=I^{\prime}(0) e^{r t}
$$

Teóricamente los precios del monopolio tratan en el punto máximo de la curva inversa de la demanda; para esto, utilizando la función de la demanda lineal $q(t)=a-b p(t)$; siendo cada una de estas variables decreciente de la otra, se desprende:

$$
p(t)=\frac{a}{b}-\frac{q(t)}{b} \ldots
$$

En este caso, para poder calcular la curva de la demanda, primero se debe de transformar los precios de la iError! No se encuentra el origen de la referencia. en precios reales constantes, tomando como año base 1990 la tasa de inflación de Estados Unidos que fue de $5.40 \%$, quedando la nueva tabla ajustada de la siguiente manera:

\begin{tabular}{|c|c|c|c|c|c|c|c|c|}
\hline \multirow[t]{2}{*}{ Año } & $\begin{array}{c}\text { Precio } \\
\text { exportación } \\
\text { del petróleo }\end{array}$ & Costo & Renta & \multirow{2}{*}{$\begin{array}{c}\text { Tipo de } \\
\text { cambio } \\
\text { Sucres por } \\
\text { US\$ }\end{array}$} & \multirow{2}{*}{$\begin{array}{c}\text { Producción } \\
\text { pozo A0045 } \\
\text { (BDP } \\
\text { promedio) }\end{array}$} & \multirow[t]{2}{*}{$\begin{array}{l}\text { Total } \\
\text { Barriles } \\
\text { Anual }\end{array}$} & \multirow[t]{2}{*}{ Beneficios } & \multirow[t]{2}{*}{$\begin{array}{c}\text { Beneficios } \\
\text { descontados }\end{array}$} \\
\hline & \multicolumn{3}{|c|}{ US\$ por barril } & & & & & \\
\hline 1975 & 11,61 & 0,62 & 10,99 & 25 & 114,00 & $37.620,00$ & $\$ 413.443,80$ & $\$ 369.146,25$ \\
\hline 1976 & 11,60 & 0,64 & 10,96 & 25 & 114,00 & $37.620,00$ & $\$ 412.315,20$ & $\$ 328.695,15$ \\
\hline 1977 & 13,10 & 0,66 & 12,44 & 25 & 100,00 & $33.000,00$ & $\$ 410.520,00$ & $\$ 292.200,03$ \\
\hline 1978 & 12,59 & 0,67 & 11,92 & 25 & 90,00 & $29.700,00$ & $\$ 354.024,00$ & $\$ 224.988,65$ \\
\hline 1979 & 23,64 & 0,88 & 22,76 & 25 & 85,50 & $28.215,00$ & $\$ 642.173,40$ & $\$ 364.386,43$ \\
\hline 1980 & 35,43 & 1,02 & 34,41 & 25 & 82,08 & $27.086,40$ & $\$ 932.043,02$ & $\$ 472.202,00$ \\
\hline 1981 & 34,61 & 1,29 & 33,32 & 25 & 73,87 & $24.377,76$ & $\$ 812.266,96$ & $\$ 367.428,32$ \\
\hline 1982 & 32,94 & 2,16 & 30,78 & 31 & 62,79 & $20.721,10$ & $\$ 637.795,33$ & $\$ 257.594,84$ \\
\hline 1983 & 38,19 & 3,62 & 34,57 & 50 & 54,44 & $17.965,19$ & $\$ 621.056,63$ & $\$ 223.959,25$ \\
\hline 1984 & 27,52 & 3,71 & 23,81 & 71 & 46,38 & $15.306,34$ & $\$ 364.444,00$ & $\$ 117.341,22$ \\
\hline 1985 & 25,95 & 4,33 & 21,62 & 93 & 38,82 & $12.811,41$ & $\$ 276.982,65$ & $\$ 79.625,89$ \\
\hline 1986 & 12,80 & 4,75 & 8,05 & 132 & 31,91 & $10.530,98$ & $\$ 84.774,37$ & $\$ 21.759,47$ \\
\hline 1987 & 16,30 & 5,69 & 10,61 & 190 & 27,13 & $8.951,33$ & $\$ 94.973,62$ & $\$ 21.765,50$ \\
\hline 1988 & 12,69 & 4,27 & 8,42 & 331 & 25,77 & $8.503,76$ & $\$ 71.601,70$ & $\$ 14.651,13$ \\
\hline 1989 & 16,21 & 3,52 & 12,69 & 532 & 23,19 & $7.653,39$ & $\$ 97.121,49$ & $\$ 17.743,73$ \\
\hline \multirow[t]{2}{*}{1990} & 20,36 & 2,06 & 18,30 & 776 & 20,87 & $6.888,05$ & $\$ 126.051,30$ & $\$ 20.561,70$ \\
\hline & & $\begin{array}{l}\text { Reserva } \\
\text { probada }\end{array}$ & & & 990,76 & $326.950,71$ & & \$3.194.049,56 \\
\hline
\end{tabular}

Tabla 2: producción petrolera pozo A0045precios reales

Fuente: datos sobre información petrolera del Ecuador Elaboración: autores

Una vez obtenidos los precios reales a 1990 se puede realizar el cálculo de la curva de demanda de la expresión 2, misma que en este caso se realiza de dos formas distintas, una a través de Excel, con la herramienta de gráficas pudiendo insertar la línea de tendencia o de forma manual con un cálculo matemático sencillo para descubrir la ecuación conociendo dos puntos:
Fórmula de la pendiente:

$$
m=\frac{P 2-P 1}{Q 2-Q 1}
$$

Resolviendo:

$$
m=\frac{20.36-11.61}{6888-37620}
$$




$$
\begin{aligned}
& m=-\frac{8.75}{30732} \\
& m=-0.00028
\end{aligned}
$$

Luego se utiliza la fórmula para la búsqueda de la ecuación con puntos:

$$
P-P 1=m(Q-Q 1)
$$

Donde m, Q1 y P1; por lo que queda de la siguiente manera:

$$
P-11.61=-0.00028(Q-37620)
$$

Resolviendo:

$$
\begin{gathered}
P-11.61=-0.00028 Q+10.71 \\
P-11.61-10.71=-0.00028 Q \\
P-22.32=-0.00028 Q
\end{gathered}
$$

\section{Resultados}

Para la elaboración del modelo se utilizó una tasa de descuento social del 12\%, y utilizando la fórmula de la demanda lineal para mayor facilidad, siendo $a=78396,91 ; b=3.512,22$ y $R=326950,71$.Donde en el primer momento con la base de datos se estimó el valor promedio de los beneficios anuales durante los 16 años de producción del pozo, se pudo determinar que la producción es decreciente, se muestran los resultados obtenidos sin la aplicación del modelo de Hotelling en la iError! No se encuentra el origen de la referencia..

Los beneficios descontados fueron de \$3176457.35 (dólares americanos), que representa una cifra significativa.Para la aplicación del modelo se utilizó la siguiente función inversa de la demanda: (esquema monopolístico), expresión 2

Para calcular la ecuación de la demanda inversa indicada en la expresión 2, se utilizó la iError! No se encuentra el origen de la referencia., dando como resultado:

$$
p(t)=22.32-0.00028 q(t)
$$

Una vez que se tiene la ecuación de la demanda inversa se pueden realizar los cálculos en Excel para

$$
Q=-\frac{1}{0.00028} P+\frac{22.32}{0.00028}
$$

Ecuación de la Demanda:

$$
Q=-3512.22 P+78396.91
$$

La RH corresponde al ingreso total menos el costo marginal; es decir la sumatoria de todos los ingresos percibidos por la venta de la extracción del recurso natural menos todos los costos marginales que incidieron en la extracción el recurso natural no renovable.

Para encontrar la maximización de los beneficios al valor presente de la extracción del petróleo se debe:

$$
\frac{p(t)-C^{\prime}(q(t))}{(1+r)^{2}}-\lambda=0
$$

encontrar el horizonte de agotamiento del recurso natural no renovable Tm:

$$
\frac{a}{2} T m-\frac{a}{2 r}+\frac{a e^{-r t m}}{2 r}=R(4)
$$

Utilizando la expresión 4, se obtiene preliminarmente un $\mathrm{Tm}=15,35$, cabe recalcar que en este resultado no se utiliza un esquema de optimización; por lo que no se conoce con certeza si en ese año se va a maximizar la función objetivo, pero si es un dato importante para conseguirlo ya que se utiliza como herramienta en el Excel a través del Solver. Donde se calcula $\mathrm{Tm}=14.93$ y $\lambda=3.992$, con los cuales no se está tan lejos de los cálculos supuestos.

Los beneficios se maximizarán en el nivel de producción, cuando los ingresos marginales sean igual al coste marginal. Como se puede evidenciar en la Tabla 3, los cálculos realizados no revelan una optimización en el momento que la reserva es negativa. 
Tabla 3: cuadro Inicial de Extracción

\begin{tabular}{|c|c|c|c|c|c|}
\hline \multirow[t]{2}{*}{$t$} & Precios & $\begin{array}{c}\text { Cantidad a } \\
\text { extraer }\end{array}$ & Beneficios & $\begin{array}{c}\text { Beneficios } \\
\text { descontados }\end{array}$ & $\begin{array}{c}\text { Reserva en el } \\
\text { tiempo }\end{array}$ \\
\hline & pm & qm & $\mathrm{Bm}$ & $\mathrm{Bm} /(1+\mathrm{r}) \mathrm{t}$ & $\mathbf{R}-\sum \mathbf{q}$ \\
\hline 0 & 13,02 & $32.667,06$ & $425.331,68$ & $425.331,68$ & $294.283,65$ \\
\hline 1 & 13,26 & $31.834,33$ & $422.037,11$ & $376.818,85$ & $262.449,32$ \\
\hline 2 & 13,52 & $30.895,42$ & $417.848,90$ & $333.106,58$ & $231.553,90$ \\
\hline 3 & 13,83 & $29.836,81$ & $412.524,63$ & $293.626,88$ & $201.717,09$ \\
\hline 4 & 14,17 & $28.643,23$ & $405.756,16$ & $257.865,38$ & $173.073,85$ \\
\hline 5 & 14,55 & $27.297,48$ & $397.151,76$ & $225.354,57$ & $145.776,38$ \\
\hline 6 & 14,98 & $25.780,14$ & $386.213,41$ & $195.667,73$ & $119.996,24$ \\
\hline 7 & 15,47 & $24.069,34$ & $372.308,04$ & $168.413,25$ & $95.926,90$ \\
\hline 8 & 16,02 & $22.140,43$ & $354.630,86$ & $143.229,46$ & $73.786,47$ \\
\hline 9 & 16,64 & $19.965,59$ & $332.158,76$ & $119.779,78$ & $53.820,88$ \\
\hline 10 & 17,33 & $17.513,46$ & $303.591,12$ & $97.748,21$ & $36.307,42$ \\
\hline 11 & 18,12 & $14.748,69$ & $267.274,52$ & $76.835,04$ & $21.558,74$ \\
\hline 12 & 19,01 & $11.631,42$ & $221.107,09$ & $56.752,68$ & $9.927,32$ \\
\hline 13 & 20,01 & $8.116,71$ & $162.416,78$ & $37.221,73$ & $1.810,61$ \\
\hline 14 & 21,14 & $4.153,88$ & $87.806,76$ & $17.967,00$ & $-2.343,28$ \\
\hline 15 & 22,41 & $-314,19$ & $-7.041,15$ & $-1.286,39$ & $-2.029,09$ \\
\hline 15,35 & 22,90 & $-2.029,09$ & $-46.463,86$ & $-8.154,91$ & $11.956,41$ \\
\hline Suma & & $326.950,71$ & & $816.277,53$ & \\
\hline
\end{tabular}

Elaboración: Autores

Para dar una solución de optimización se utiliza la herramienta SOLVER del Excel y de esta manera encontrar que $\mathrm{T}_{\mathrm{m}}=14.86$ y $\lambda=3.989$, donde la sumatoria de extracción coincide con el con la $\Sigma q c(t)$.

\begin{tabular}{|c|c|c|c|c|c|}
\hline \multirow[t]{2}{*}{$\mathbf{T}$} & Precios & $\begin{array}{c}\text { Cantidad a } \\
\text { extraer }\end{array}$ & Beneficios & $\begin{array}{c}\text { Beneficios } \\
\text { descontados }\end{array}$ & $\begin{array}{c}\text { Reserva en el } \\
\text { tiempo }\end{array}$ \\
\hline & pm & Qm & $\mathrm{Bm}$ & $\mathrm{Bm} /(1+\mathrm{r}) \mathrm{t}$ & $R-\sum q$ \\
\hline 0 & 13,04 & $32.607,89$ & $425.110,63$ & $425.110,63$ & $294.342,81$ \\
\hline 1 & 13,28 & $31.767,62$ & $421.756,11$ & $376.567,96$ & $262.575,20$ \\
\hline 2 & 13,55 & $30.820,21$ & $417.491,67$ & $332.821,81$ & $231.754,99$ \\
\hline 3 & 13,85 & $29.752,01$ & $412.070,51$ & $293.303,65$ & $202.002,98$ \\
\hline 4 & 14,19 & $28.547,62$ & $405.178,86$ & $257.498,49$ & $173.455,36$ \\
\hline 5 & 14,58 & $27.189,67$ & $396.417,86$ & $224.938,14$ & $146.265,69$ \\
\hline 6 & 15,02 & $25.658,59$ & $385.280,45$ & $195.195,07$ & $120.607,10$ \\
\hline 7 & 15,51 & $23.932,30$ & $371.122,02$ & $167.876,75$ & $96.674,80$ \\
\hline 8 & 16,06 & $21.985,91$ & $353.123,13$ & $142.620,51$ & $74.688,89$ \\
\hline 9 & 16,69 & $19.791,37$ & $330.242,06$ & $119.088,60$ & $54.897,52$ \\
\hline 10 & 17,39 & $17.317,02$ & $301.154,51$ & $96.963,69$ & $37.580,50$ \\
\hline 11 & 18,18 & $14.527,21$ & $264.176,99$ & $75.944,57$ & $23.053,29$ \\
\hline 12 & 19,08 & $11.381,70$ & $217.169,35$ & $55.741,96$ & $11.671,59$ \\
\hline 13 & 20,09 & $7.835,16$ & $157.410,93$ & $36.074,52$ & $3.836,43$ \\
\hline 14 & 21,23 & $3.836,43$ & $81.443,09$ & $16.664,87$ & $-0,00$ \\
\hline 14,85832 & 22,32 & 0,00 & 0,00 & 0,00 & 0,00 \\
\hline Suma & & $326.950,71$ & & $2.816 .411,23$ & \\
\hline
\end{tabular}

La reserva se agota en el período 14,85 años aproximadamente. Los beneficios descontados son de \$2.816.411,23(dólares americanos), cifra que para efectos de esta aplicación es inferior a la obtenida.Pero, a pesar de haber obtenido mayores ingresos no fueron bien utilizados ya que la deuda 
pública superaba la inversión, desfavoreciendo a las personas de las zonas costeras de extracción del recurso natural (Estrada, 2011).

\section{Conclusiones}

La extracción petrolera analizada en la presente investigación deja como resultado que las empresas extranjeras fueron las que se beneficiaron, ya que en los años considerados de mayor demanda las políticas públicas no determinaban regalías y rentas en beneficio del Estado y de la sociedad, solamente entregaban "beneficios" a los trabajadores de las mencionadas empresas, de tal manera que en las áreas de incidencia la situación socio- económica no ha mejorado.

Por tener un modelo teórico de aplicación con la regla de Hotelling y la falta de información sistematizada, hubo que realizar simulaciones para llegar a un modelo aceptable, también se realizó entrevistas a expertos en petróleo.

El gobierno actual se ha preocupado de renovar contratos con las empresas petroleras transnacionales, exigiendo mayor participación del Estado, cuando los precios por barril pasan un determinado umbral; esa diferencia aumenta los ingresos presupuestarios del gobierno.Las regalías, la renta del petróleo, los pozos que mantiene el Estado como dueño del recurso natural, han permitido financiar casi el 50\% del presupuesto del erario nacional, representando entre el 20 y $25 \%$ de las exportaciones totales.Para el presupuesto del 2016, el Estado no considera los ingresos petroleros como parte del presupuesto general para pagos corrientes, sino para inversión de bienestar social.

Los ingresos petroleros del Ecuador en el periodo de gestión del actual gobierno, que ha recibido ingresos extraordinarios por más de 300 millones de dólares, de alguna manera se han utilizado para mejorar el capital natural, obras de infraestructura: centrales hidroeléctricas, mejorar carreteras; en el área de la salud, nuevos hospitales, mejorar la atención, pero en el área de la generación de trabajo, hubo una debilidad, lo que se denomina depreciación del capital humano, por cuanto la tasa de desempleo juvenil es muy alta, además a nivel de los profesionales, también se repite el mismo fenómeno.

Hasta el año 2000 la inversión petrolera estaba en manos del sector privado. A partir de 2007 se revierten las concesiones al Estado, siendo el mayor accionista del área petrolera, por eso se refleja la inversión del periodo 2007 al 2014 que se incrementa del 698.4 a 4000 mil millones de dólares. A esto se suma que el gobierno fija políticas petroleras y los recursos que se llevaban las empresas transnacionales pasaron a ser administrados por el Estado.

La inversión pública crece de igual manera en función al precio del petróleo, en tanto que la privada se ha mantenido más o menos constante (estacionaria).Porque estos cambios están en función del precio del petróleo, además de políticas internas en que los recursos naturales no renovables sean manejados por el Estado tal como lo estipula la Constitución del Ecuador.

\section{Referencias bibliográficas}

Acosta, A. (2004). El petróleo en el Ecuador: Dimensiones y conflictos.

Asheim, G., \& Buchholz, W. (2000). The Hartwick rule: Myths and Facts. Department of Economics, University of Oslo, 1-30.

Baquero Daniel y Mieles José. (2015). Los booms petroleros ¿Qué cambió en los últimos 40 años? Carta Económica.

Daly, H., \& Farley, J. (2004). Ecological economics: Principles and applications. Washington: Islandpress.

Estrada, J. (2011). Ancón, 100 años en la historia petrolera del Ecuador.

Figueroa, E. (2006). El Comportamiento Económico del Mercado del Petróleo. España: Diaz de Santos.

Hartwick, J. M. (1977). Hartwick, J. M. (1977). Intergenerational equity and the investing of rents from exhaustible resources. The American Economic Review.

Harwick, J. M., \& Olewiler, N. D. (1998). The Economics of Natural Resource Use (Second ed.). United States of America: Addison-Wesley.

Hotelling, H. (1931). The economics of exhaustible resources. . The Journal of Political Economy, (39(2)), 137-175. 
Ministerio de Finanzas. (2016, Octubre 5). MINISTERIO DE FINANZAS. Retrieved from MINISTERIO DE FINANZAS, Sitio Web:

http://www.finanzas.gob.ec/wpcontent/uploads/downloads/2016/04/PRE SENTACION-INFORMACI\%C $3 \% 93 \mathrm{~N}-$ T\%C3\%89CNICA-20152.pdf

Ministerio de Hidrocarburos. (2016, Octubre 5). MINISTERIO DE HIDROCARBUROS. Retrieved from http://www.hidrocarburos.gob.ec/wpcontent/uploads/2016/03/Dise $\% \mathrm{C} 3 \% \mathrm{~B} 1$ o -Rendici\%C3\%B3n-de-Cuentas.pdf

Pareja Yannuzzelli, C. (2016, Octubre 5). MINISTERIO DE HIDROCARBUROS. Retrieved from http://www.hidrocarburos.gob.ec/wpcontent/uploads/2016/04/INFORME-DE-
RENDICION-DE-CUENTAS-2015MINISTERIO-DE-HIDROCARBUROSCPCCS.pdf

Perman, R. M., MacGilvary, J., \& Common, M. (1999). Natural Resource and Environmental Economics (Segunda ed.). Longman.

Romero, C. (1997). Economía de los recursos ambientales y naturales (Segunda ed.). España: Alianza Economia.

Velasteguí, L. A. (2004). La era petrolera en el Ecuador y su incidencia en el presupuesto general.

Vincent, J. R., \& Mohamed Ali, R. (1997). Enviroment and Develpment in a Resource-Rich Economy.United States: Harvard University Press. 